\title{
Paraplegia
}

\section{Understanding and Treating Motivation Difficulties in Ventilator-dependent SCI Patients}

\author{
S. A. Jordan, PhD, ${ }^{1}$ W. R. Wellborn III, MA, PhD, ${ }^{2}$ J. Kovnick, BS, PG-4, \\ R. Saltzstein, $\mathbf{M D}^{4}$ \\ ${ }^{1}$ Department of Psychology, Madonna Centres, 5401 South Street, Lincoln, NE \\ 68506-2134, ${ }^{2}$ Department of Psychology, California School of Professional \\ Psychology, Fresco, CA, ${ }^{3}$ Medical College of Wisconsin, ${ }^{4}$ S pinal Injury Unit, Zablocki \\ VA Medical Center, Milwaukee, WI, USA.
}

\begin{abstract}
Summary
Motivation for spinal cord injury rehabilitation is a difficult management task for clinicians. Ventilator-dependent quadriplegics experience conditions which are particularly likely to lead to motivational problems. The following article provides a theoretical model with which to understand patient motivation. The model conceptualises patient motivation as a patient-rehabilitation environment fit problem and employs concepts from social learning theory to facilitate understanding and intervention with such difficulties. The model is applied to two ventilator-dependent rehabilitation cases. Recommendations for clinical practice are provided.
\end{abstract}

Key words: Motivation; Ventilator-dependent quadriplegia; Quadriplegics; Spinal injury; Social learning theory.

Patient motivation and compliance in rehabilitation settings have proved to be complex issues for health care professionals. This general statement applies particularly to the rehabilitation of the ventilator-dependent patient with spinal cord injury (SCI). Many of the personal characteristics of this population in combination with situational contingencies in such rehabilitation settings interact to enhance the potential for 'motivation' difficulties. Not only is there conceptual disagreement among scholars regarding appropriate constructs to apply to this topic, there is little practical advice for clinicians who deal with these matters on a daily basis.

The following article aspires to provide a framework with which clinicians can conceptualise patient motivation; this framework can be used to derive program approaches which may increase the likelihood of positive rehabilitation outcomes. In order to achieve this goal, we will: (a) provide a definition of motivation that includes patient characteristics and milieu variables; (b) discuss some unique contingencies operating in SCI rehabilitation settings; (c) provide a conceptual model which includes some variables that are thought to be necessary to understand moti- 
vation; (d) apply this model to two cases of quadriplegia; and (e) outline approaches we have found useful in tailoring rehabilitation to patient characteristics.

Before we present our conceptual model of motivation and relate it to clinical suggestions, we would like to present two cases of quadriplegia that represent extremes of rehabilitation outcome and patient motivation. After presenting these cases, this paper will explore some factors that contribute to successful rehabilitation of the ventilator-dependent SCI patient.

\section{Presentation of two ventilator-dependent cases of SCI}

Following are the cases of two individual patients who sustained high cervical cord injury requiring ventilator support. Their progress exemplifies the extremes of success and failure from a rehabilitation perspective.

\section{Case 1}

Nature of injury. This is a ventilator-dependent 45 -year-old man who experienced a C1-2 subluxation after a horseback riding accident in 1982 with a resulting C2 motor and sensory incomplete quadriplegia. The injury has left him with a patchy sensory input and trace motor functioning of his hands and feet on one side.

Course of treatment. The patient underwent his rehabilitation care in an interdisciplinary setting and since the time of his discharge has returned to an independent living situation. The initial care demonstrated active and creative input by the patient in the planning of therapeutic approaches and discharge outcomes. His medical course was relatively uncomplicated with his status at discharge requiring ventilatory support. He had virtually no medical complications during his initial hospitalisation and since that time had required only routine support. Since the time of his initial hospitalisation, he has been an advocate for any and all treatment approaches that have been offered and provided.

Employment. This patient was employed before his injury in the sale of communication devices. Since his injury he has returned to this vocation and now employs several full-time caregivers who are able to assist him in transportation around his community where he engages in sales to business and industry. His financial resources are satisfactory and appear not be be a major impediment to his ongoing support.

Social situation. Divorce proceedings were initiated prior to his injury, and were completed subsequently. Since the time of injury, he has received ongoing support from family and friends including his mother, daughter, and his church group.

Living situation and medical support. At the time of his injury, he was remodelling his home; this was completed following his injury. He lives near a major city and he receives ongoing medical support nearby. In addition, he receives his annual and semi-annual spinal cord injury care at a regional VA Hospital. 


\section{Case 2}

Nature of injury. This is a ventilator-dependent 48 -year-old man who experienced a C4 incomplete SCI as a result of a motor vehicle accident; he was believed to be intoxicated at the time of the accident.

Course of treatment. He underwent his rehabilitation care at his regional VA Medical Center. During the course of hospitalisation, he had multiple medical complications including pneumonia lung abscess, pressure sore, and renal calculi. He had a limited dialogue with the caregiving team while initially hospitalised though he was noted to have extended interactions with both his brother and friends from his farm community, and he seemed to have very few independent comments about his anticipation of the future. Despite a level of injury that allowed ventilatory independence, he continued to select constant mechanical support of ventilation. Thus, discharge from rehabilitation was to a long-term care facility that was both relatively close to his former residence and was capable of assisting in his ventilatory support management.

Employment. This patient has a high school education and was employed as a farmer. Following the injury, he and his brother sold their farm, having little financial support.

Social situation. Single, and never married prior to his injury. His primary support was his brother with whom he lived. He reported that he seldom left the farm, and did not participate in community activities. Post-injury, his contact with his brother was further limited by his placement in long-term nursing care.

Living situation and medical support. He was living in a remote rural setting, prior to injury. The combination of his need for pulmonary support and the relative isolation of his prior living situation resulted in his placement in a long-term care facility.

\section{Motivation}

The approach of this paper is to consider motivation as an interactive force between patients and the treatment environment. This approach defines motivation as a relationship rather than as a characteristic of the patient or of the rehabilitation setting. 'Motivated' patients are those whose personal characteristics and resources are well-matched to the demands of the treatment environment (high personenvironment fit). In this formulation, 'unmotivated' patients reflect a mismatch of treatment demands on patients who are not responsive to those demands (low or poor person-environment fit).

This interactive definition of motivation assumes that patients vary in their personal coping resources. Further, it assumes that rehabilitation settings and professionals are capable of responding flexibly to patient needs. Finally, it asserts that higher levels of flexibility and responsivity of clinical approaches to the variability 
among patients will increase the likelihood of 'person-environment fit', and will result in more frequent rehabilitation successes.

Patients have restrictions on their ability to respond flexibly to environmental demands; these restrictions can result from a lack of experience, personality disturbances, limited intelligence, and other characteristics which can impede patients' abilities to function adaptively in the rehabilitation setting.

Similarly, clinicians and treatment environments have contingencies that restrict their ability to respond flexibly to patient needs. We will restrict ourselves to discussion of the restrictions that occur among clinicians.

\section{Limitations of clinicians flexibility toward patients}

One of the more important limiting factors among physiatrists and other rehabilitation professionals is the tension that obtains from paradigm conflicts between the 'medical' model and the 'learning/rehabilitation' model. Trieschmann (1988) notes the distinction between the medical model of rehabilitation and the educational or learning approach. In the medical model, the patient is a passive recipient of treatment and is often not given any choices in the process of rehabilitation. Treatment content focuses on mobility and activities of daily living (ADL) training. Anxiety, lack of motivation, and depression are treated with counselling. Progress is evaluated by how well the patient meets the goals set down for him or her by the rehabilitation team.

Conversely, the learning approach is viewed as the process of teaching the individual how to live with the disability in his or her environment (Fordyce, 1975; Treischman, 1988). The person is assumed to be healthy but in need of education which will facilitate return to the world outside the hospital, viz., the individual should know how to solve unique problems related to the environment by making independent decisions, adapting to circumstances, and at times changing the environment (Friedan and Cole, 1985). In this model, the team assesses the impact of the disability on the individual and takes into account the environment to which he or she will return. Such programmes are designed to help the patient achieve success and reward from life in a world dominated by and designed for the nondisabled individual. Training content focuses on ADL's, mobility, social skills, sexuality, as well as on redefinitions of productivity, family roles and economic survival. Of course, the individual's family (if any) will be involved since they will be an integral part of the environment upon discharge.

In summary, the learning approach focuses on the behaviour of the individual with the spinal injury. His or her behaviour is the primary outcome by which the success or failure of the efforts can be judged. The injured person must be an active participant in the process. The rehabilitation program is designed by the staff, with, not for, the person to meet his needs as they relate to his personality and to the environment from which he comes and to which he will return (Trieschman, 1988).

It is often assumed that management of the person with SCI occurs in two fairly discreet stages: acute management and rehabilitative cares and training. Immediately after injury, hospital personnel are concerned with the patient's survival and creation of medical stability with maximal preservation of bodily functions. This can include operative management, treatment of associated injuries, bowel and 
bladder management, ventilatory support (if needed), and attention to maintenance of skin integrity (Drayton-Hargrove and Reddy, 1986). After the patient is medically and surgically stable, the rehabilitative stage is presumed to begin. In this stage, the physiatrist and rehabilitation team begin the process of working with the patient to gain maximum mobility, optimal independence in self cares, ADL's, and skills necessary to reintegrate into society and family.

In fact, Keith $(1969,1971)$ has proposed a 'pacemaker' model of rehabilitation that makes these two stages of treatment discreet, adapting the treatment approach to the patient's stage of rehabilitation.

Unfortunately, many SCI patients require continuous 'acute' medical management throughout their rehabilitation. Such ongoing medical needs include spasticity, bowel and bladder management, care and management of the integument, and pulmonary status. This mixture of patient medical needs (which are most efficiently served through traditional medical approaches) and rehabilitation needs (which are most successfully approached through a learning approach) creates a dilemma for physicians, who are expected to examine which type of approach is most appropriate for a specific problem.

For example, pain management is a notoriously difficult treatment issue for physicians, and one that easily illustrates this dilemma. Pain is usually treated pharmacologically during the acute stages. Unfortunately, this approach is less effective for chronic pain. Most researchers agree that chronic pain is a complex interaction of physical traumata and psychology that requires an integrated treatment approach, including physician supervision and patient education and treatment. Not only is it difficult for physicians to determine the differential between acute and chronic pain, but it is also a challenge to encourage the patient to evolve from a passive recipient of pain treatment (medical model) to an active consumer of services (rehabilitation model). Furthermore, many patients require ongoing medical supervision for their pain symptoms, thereby extending the physician's dilemma over time.

\section{A proposed 'motivation' model}

Our person-environment fit model of motivation requires an understanding of both patient variables and treatment milieu variables. A complete model would include such patient variables as: physical health functioning (historical and current), social history (including education, occupation, social status, social support, family history and living situation), intelligence and neuropsychological status, and personality. Treatment milieu variables would include: treatment philosophy (medical versus rehabilitation model), inpatient versus outpatient treatment setting, personnel, organisational structure (hierarchical versus team), size of program, etc. Variables which have been considered predictors of outcome are age, educational level, pre-injury vocational achievement, social adaptability, family support, marital status, and level of injury (Drayton-Hargrove and Reddy, 1986; Woolsey, 1985).

This article will emphasize two sets of variables within this domain: patient personality and physician management of treatment. We will employ principles from social learning theory (SLT) to describe patient responses to the rehabilitation environment. It is anticipated that these principles will provide a framework with 
which physicians may understand and appropriately address difficulties in motivation among rehabilitation patients.

One of the more familiar social learning constructs is locus of control (LOC). LOC refers to individuals' characteristic beliefs about the source of their reinforcements. Rotter (1966) distinguised between people who have an internal locus of control and those having an external locus of control. Internals feel that their own actions have a direct influence on their fate in life. Externals, on the other hand, believe that life's punishments and rewards occur as the result of chance, fate, or the influence of others. Levenson (1974) extended Rotter's conception into a threedimensional construct, including: Internality, Powerful Others, and Chance. Wallston, Wallston, and De Vellis (1978) developed a scale to measure these constructs. In brief, each of these three constructs may vary from low levels to high levels. For example, a patient may have an internal LOC, a relatively strong belief that treatment staff are influential in the patient's outcomes, and a relatively weak anticipation that chance or luck influences his life. Other patients may vary on each of these three dimensions, thereby influencing their approach to rehabilitation. This multidimensional view of LOC differentiates between two types of external control: 'belief in the basic unordered and random nature of the world and belief in the basic order and predictability of the world, coupled with the expectancy that powerful others are in control' (Levenson, 1981).

It is critical to understand that it is not our perception of the relationship between a particular patient's behaviour and outcome that is important; the critical issue is what the patient expects and believes. The extent to which patients have an internal or external locus of control can affect how they respond to the rehabilitation setting. Persons with an external locus of control believe that their behaviour has little effect on what they get out of life; therefore they might tend to be less persistent in achieving goals. Fortunately, we have observed that many patients (who often enter rehabilitation with an external LOC and concomitant feelings of helplessness) tend to develop a more internal locus of control as they begin to experience successes as the rehabilitation program proceeds.

A second SLT construct that is useful for this motivation model is outcome expectancy. Bandura (1977) defined outcome expectancy as 'a person's estimate that a given behaviour will lead to certain outcomes'. Rotter (1954) separated outcome expectancy into two types, which can be thought of as specific expectancies and generalized expectancies. Specific expectancies relate a behavioral response to proximate outcomes (i.e., 'If I increase my UE strength, I will be able to make independent transfers'). The generalised expectancies relate a behavioural response to distant outcomes (i.e., 'If I make independent transfers, I will have a high quality of life').

Two other variables are of interest in this model of motivation: outcome value and change cost. Outcome value is simply the importance or value that a patient places on a given rehabilitation outcome. Change cost refers to the amount of physical, psychological, or practical costs associated with changing a behaviour. Both of these constructs are subjective in nature, and they may or may not closely correspond with objective data. Thus, some outcomes may be more important for some patients than for others; similarly, a behavioural change may be inconsequential for some patients, while it may seem an insurmountable barrier for others.

In general, the SLT constructs highlight the importance of the patient's subjec- 
tive experience of the rehabilitation environment as it relates to his life. Ignorance of these considerations on the part of treatment providers often leads to inappropriate outcome goals and patient-provider tension that is usually subsumed under the headings 'poor motivation' and 'negative outcome'.

\section{Application of the model to the case studies}

The purpose of this section is to apply SLT constructs to the two previously described cases in an effort to illustrate the utility of SLT constructs for understanding patient motivation during rehabilitation of ventilator-dependent patients. It should be understood that the authors applied these constructs to the patients' cases from their viewpoint, retrospectively. A more appropriate method of applying these constructs would be obtaining the patients' actual experiences through an interview or other measurement strategy that assesses their attitudes directly.

The cases presented earlier in this paper are examples of two very different people. They entered the rehabilitation programs with the same goal: to get better. How they reacted to their situations was far from the same. Case 1, the C2 ventilator dependent quadriplegic, is now self-reliant in terms of economics, social life and direction of his cares. He had achieved success prior to his injury and appears to have had a pre-morbid personality of being independent and expecting success. He was a healthy religious man at the time of injury.

On the other hand, case 2 is a $\mathrm{C} 4$ quadriplegic who is also ventilator dependent. With his level of injury, he need not be ventilator supported full-time, but his medical problems and personality have compromised him to such a degree that he had resisted efforts toward breathing on his own. That he was an alcoholic and had chronic lung disease prior to the injury certainly were factors that hampered his progress. He had neither friends nor family for support and most likely has very little understanding of why he should do what he is told to do by the therapists.

\section{Locus of control}

Case 1 is probably what Wallston et al. (1978) would refer to as a 'Believer in Control'. He would likely have scored high on the Internality and Powerful Other LOC subscales, and low on the Chance subscale. He behaved as if both he and his physicians controlled his rehabilitation. He took a very active role and he benefited from patient education. Premorbidly, his work involved the sale of communication devices; this work required self-directed effort and independent decisionmaking skills.

Case 2 would be described as a 'Pure External' by Wallston (1978), since he took a very passive role during rehabilitation. He probably would have scored low on the Internality LOC subscale, and high on the Powerful Other and Chance subscales. He tended not to behave proactively, and he believed that he had little influence in controlling his rehabilitation outcome. His occupation was farming which requires self-directed behavior, but is heavily dependent on weather conditions for its success. Another potential contributing factor to his 'Pure External' orientation was his multiple medical complications including, pneumonia, lung abscesses, pressure sores, and renal calculi. 


\section{Outcome expectancy}

In considering outcome expectancy it appears that case 1's estimate of outcome expectancy was high and case 2's was low. This is based on each patient's premorbid functional level, education, experiential base, and social style. Case 1 had more experience with others who had overcome adversity; additionally, he was more socially involved premorbidly.

\section{Outcome value and change cost}

It is difficult to apply this construct without asking each patient about his perception of the importance of rehabilitation outcome to his lifestyle. When we considered outcome value, we estimated that case 1 would place a higher value on rehabilitation outcome when compared to case 2 . We made this judgment based upon case l's job success, effective social support system, and financial stability.

We suspect that both the patients experienced extremely high levels of perceived change cost. Each had considerable amounts of physical, psychological, and behavioural costs associated with his rehabilitation. In addition to the difficult tasks of rehabilitation, case 1 had to redesign his house to adapt to his disability; case 2 sold his farm because he was no longer able to perform the tasks of farming nor did he have financial resources to continue to manage the operation.

It is obvious these 2 patients represent opposite extremes of rehabilitation outcome, having entered their programs with widely different personalities. One cannot expect all patients to do as well as case 1 , that would be an unrealistic desire. We can, though, give our best efforts to each person. To do so, we must not be satisfied only to individualise treatment content. We must also be sensitive to the need for individualising treatment approach in order to achieve optimal interaction between patient and environment, thereby maximising rehabilitation outcome.

\section{Conclusions}

When working with SCI individuals, it is essential that one remains aware of feelings of lost control or feelings of helplessness. The onset of injury is often traumatic and the patient may not experience a sense of control of the events around him or her. Equally absent may be a sense of predictability. During successful rehabilitation, feelings of control and predictability begin to return as the patient experiences success; in some cases, the sense of control is harder to obtain, either as a result of premorbid personality or of the vagaries of medical complications.

Seligman (1975) proposed a theory of learned helplessness which states that a person, upon exposure to uncontrollable outcomes, develops expectations that future outcomes will also be uncontrollable. The major consequences of this are threefold: (1) motivational (there is a reduced motivation to initiate voluntary responses that control other events); (2) cognitive (when a person has had the experience of uncontrollability, he has trouble learning that his new response has succeeded when it actually did, and thus there is a distortion of the perception of control); and (3) emotional [initially there is a heightened state of emotionally (fear), which, with further experience with uncontrollability, changes to depression] (Treischman, 1988, p. 90). Seligman believes that to overcome this 'learned 
helplessness', persons must relearn that there is a contingency between their behaviour and environmental consequences.

When patients are not experiencing success in rehabilitation, the problem may be that they cannot envision that success. That is, patients may not have a clear understanding of or agreement with rehabilitation goals. Additionally, patients may not have a sense of confidence in their ability to reach those goals. These constitute major blocks to progress in the rehabilitation setting.

To ventilator-dependent SCI patients the tasks of rehabilitation may understandably seem insurmountable. Frequently, these patients focus on their deficits rather than what new skills and behaviour they must learn; such patients experience their lives as unpredictable and uncontrollable. It is the responsibility of the clinician to identify patient's goals and abilities, and then design approaches to achieving those goals. With individualised approaches, 'the process itself becomes an automatic reinforcement program and may produce natural competence and achievement drives far more powerful than the promise of an extrinsic reward at some point in the distant future' (Jeffery, 1981, p. 34). Such approaches encourage patients to change the way they think about their problems, enable progression from subgoal to subgoal, and enhance patients' feelings of being in control (Hooker, 1986; Jeffrey, 1981).

A common trap for rehabilitation professionals relates to the influence of the 'medical model' on their treatment approaches. It is easy to see how the clinician, who has undergone years of training in 'knowing' what is 'best,' can conclude that if only patients did what were they told, rehabilitation outcomes would be more favourable. Complications might be less frequent and the resulting medical morbidity and need for hospitalisation would decline in frequency. Unfortunately, many professionals are not trained in the art of patient collaboration and negotiation skills which are necessary to encourage patients' perceptions of control, and which are, therefore, an important foundation for rehabilitation outcome. Even among those clinicians who feel adept with these skills, there is consistent ambivalence that arises from the ambiguity related to knowing when to take control of situations ('medical model') and when to facilitate situations by acting as a consultant to patients ('learning model'). This is particularly true among ventilator-dependent patients, since medical crises are often a constant concern.

This ambivalence is increased by the high costs of reacting 'inappropriately' to situations. Medical morbidity when not taking control of situations that imminently endanger patients' health, and behavioral co-morbidity (passivity to rehabilitation and self-cares, failure to become educated about SCI, etc.) when not providing a facilitative role during situations that may ultimately endanger patients' health status after discharge.

\section{Clinical recommendations}

We would like to translate our perspectives into practical approaches to the rehabilitation of ventilator-dependent SCI patients. In overview, the purpose of our approach is to understand and encorporate patients' subjective experiences into our treatment plans. While these matters are often delegated to psychologists, social workers, and other counsellors, we believe that this perspective is useful for all rehabilitation professionals who rely on patient participation in their efforts. 
Assessment

As is true in all clinical situations, assessment is the initial step in the derivation of appropriate interventions. The following domains should be assessed in attempting to understand patients' approaches toward rehabilitation. These domains relate to the patient's LOC, outcome expectancies, reward values, and change costs constructs we have discussed above. We have provided a sampling of questions which might address the potential issues:

1. Patient's general health concerns: What concerns do you have about your health? What aspects of your health are most worrisome to you (least worrisome)?

2. Patient's knowledge, attitudes, and beliefs about SCI: What do you know about SCI? Do you know anyone with SCI? What do you think of someone who is disabled (wheelchair-bound, ventilator-dependent)? What does your situation mean for your lifestyle, friendships, family, job, etc?

3. Patient's perception of personal risk: How concerned are you about deterioration of your health? What do you see as the probability of your health improving?

4. Patient's expectations for treatment and belief in treatment effectiveness: How can we help you gain more independence? What sorts of treatment efforts do you see as being the most help for you? How independent do you think our program will let you become? In what ways will our program help you become more independent?

5. Other motives (social approval, appearance, etc).

6. History: This information is intended to gain information about past approaches (personal and familial) to other types of adversity, as well as clues related to methods of obtaining personal successes.

\section{Interventions}

The overriding goal for these interventions is to encourage and support a sense of control and predictability for these patients. In ventilator-dependent quadriplegics, nearly all physical control over the environment is lost. Therefore, verbal control becomes a higher priority and higher probability behaviour. Verbal control over the treatment team must be moulded in ways that assist patients in accomplishing the goals of rehabilitation.

1. Education: Most of rehabilitation is a form of patient education. It is, therefore, of critical importance that we obtain patients' participation in and understanding of the education process. Patient participation is encouraged by making rehabilitation efforts and goals personally relevant to each patient. Relevance is achieved by translating abstract concepts into concrete images or sensations; it is also supported by encorporating information (both content and method of presentation) into patients' belief systems and perceptions. Incorrect beliefs need to be informed, and correct information should be built upon. Relevance is also encouraged by utilizing the patient's past experience as a method of informing current needs.

2. Negotiation: The motivational importance of negotiation between clinician and patients relates to the process of giving patients verbal and behavioural control of their treatment and their fates. Negotiation extends informed consent to include soliciting patients solutions to problems, allowing patients to 'try it their way' 
under safe conditions, and being available just to listen when the patient feels frustrated.

3. Problem-solving: Problem-solving is a form of negotiation that includes: (1) setting goals; (2) generating alternative solutions; (3) selecting and implementing a solution; and (4) evaluating the outcome. This method of achieving goals is often not an obvious process for patients; many are helped to obtain a sense of control simply by learning to implement this process in a logical, methodical manner.

4. Maximising patient control: We have already mentioned this intervention in the suggestions made above. It is listed primarily to re-iterate the importance of giving patients a sense of control over their destinies by encouraging reasonable levels of independent thought and action.

5. Manipulating reward contingencies: We have found that it is often easier to alter environmental contingencies than try to change the patients' attitudes from the onset. Therapists can assist patients through graded exercises where these patients repeatedly see the fruits of their efforts.

6. Increasing attitude-functioning fit: It is often beneficial to encourage the patient to realign his or her lifestyle interests and preferences in accordance with remaining assets (Decker and Schultz, 1985: Kanellos, 1985).

7. Adapting rehabilitation approach to patient personality: It is important for the rehabilitation team to respect individual differences in patient personality. For example, some 'difficult' patients have a very strong internal locus of control (insist on doing things their own way). Rather than countering such patients, it is often more helpful to involve these people more in defining their own schedule, setting their rehabilitation goals and structuring ways to reach these goals.

Other 'difficult' patients may have an exaggerated external locus of control and might succumb easily to learned helplessness and appear highly unmotivated. Such patients may require much more structure and be very receptive to receiving specific rewards for particular behaviors (Treischman, 1988).

\section{References}

BANDURA A 1977 Self-efficacy: Toward a unifying theory of behaviour change. Psychological Review 84: 191-215.

DeCKER SD, SChUlz R 1985 Correlates of life satisfaction and depression in middle-aged and elderly spinal cord injured persons. American fournal of Occupational Therapy 39:740-745.

DRAYTON-HARGROVE S, REDDY MA 1986 Rehabilitation and long-term management of the spinal cord injured adult. Nursing Clinics of North America 21:(4):599-610.

FORDYCE W 1975 Research on influencing level of patient participation in the rehabilitation process. Selected research topics in spinal cord injury rehabilitation. Grant No. 16-P-56813/6-13, Houston. Texas Institute for Rehabilitation and Research.

FRIEDAN L, COLE JA 1985 Independence: The ultimate goal of rehabilitation for spinal-cord injured persons. American fournal of Occupational Therapy 39:(11):734-739.

HOOKER EZ 1986 Problems of veterans spinal cord injured after age 55: Nursing implications. Fournal of Neuroscience Nursing 18:(14):188-195

JEFFREY, DL 1981 Cognitive clarity: Key to motivation in rehabilitation. Fournal of Rehabilitation 47(3):33-34.

KANELLOS MC 1985 Enhancing vocational outcomes of spinal cord injured persons: The occupational therapist's role. American fournal of Occupational Therapy 39:726-733.

LEVENSON H 1974 Activism and powerful others: Distinctions within the concept of internal-external control. Fournal of Personality Assessment 38:377-383.

LEVENSON H 1981 Differentiating among internality, powerful others, and chance. In: HM Lefcourt (ed), Research with the locus of control construct. New York: Academic, 15-63.

ROTTER JB 1954 Social learning and clinical psychology. Englewood Cliffs, NJ: Prentice-Hall. 
Rotter, J 1966 Generalized expectancies for internal versus external control of reinforcement. Psychological Monographs: General and Applied 80:1-28.

ROTTER JB 1982 Social learning theory. In: NT Feather (ed), Expectations and actions: Expectancyvalue models in psychology. Hillsdale, NJ: Lawrence Erlbaum Associates, 241-260.

Seligman, M 1975 Helplessness: On depression, development, and death. San Francisco: W.H. Freeman and Co.

Treischman RB 1988 Spinal cord injuries: Psychological, social, and vocational rehabilitation, 2nd ed. New York: Demos.

Wallston KA, WaLlSton BS, DeVellis R 1978 Development of the multidimensional health locus of control (MHLC) scales. Health Education Monographs 6:160-170.

WOOLSEY RM 1985 Rehabilitation outcome following spinal cord injury. Archives of Neurology 42: 116-119.

ZANE MD, LowENTHAL M 1960 Motivation in rehabilitation of the physically handicapped. Archives of Physical Medicine and Rehabilitation 41:400-407. 